\title{
Pneumopericardium after blunt chest trauma: A sign of severe injury?
}

\author{
Thierry C. Roth, MD, and Ralph A. Schmid, MD, Berne, Switzerland
}

$\mathrm{P}$ enetrating injuries might result in pneumopericardium, but it occurs only very rarely after blunt trauma. In some cases tension pneumopericardium has been reported, which requires an urgent decompressive procedure. Although several hypotheses of the pathogenesis of pneumopericardium are described, it is not exactly known why and under which circumstances an air tamponade occurs. We report a case after blunt trauma with associated major polytrauma, including right pneumothorax and pneumopericardium, which was treated with chest tube insertion only.

\section{Clinical Summary}

A 57-year-old white man was admitted to our institution after major polytrauma as a result of a $10-\mathrm{m}$ fall from a scaffold. The patient was intubated on site and had a Coma Glasgow Score of 8 . His past medical history disclosed treated diabetes mellitus. At admission, the patient was hemodynamically stable, with a blood pressure of $125 / 75 \mathrm{~mm} \mathrm{Hg}$, heart rate of 120 beats/min, respiratory rate of 20 breaths $/ \mathrm{min}$, and oxygen saturation of $96 \%$ with an inspired oxygen fraction of 1.0. He had hematoma of the right orbita and temporal scalp, and the pupils were symmetrically reactive, but the motility of the right arm was obviously diminished. He had contusion of the right chest without flail chest or subcutaneous emphysema, and lung auscultation was diminished on the right side. Abdominal examination did not show tenderness, but urethrorragia was observed, the pelvis was stable, and the extremities did not present any sign of fractures.

A chest radiograph showed a right-sided pneumothorax associated with pneumopericardium but no rib fractures (Figure 1), and therefore a chest tube was inserted. Cervical spine and pelvis radiography were performed and showed bilateral iliopubic and ischiopubic fracture associated with sacrum fracture on the right side, without dislocation. Laboratory examination revealed a hemoglobin level of $90 \mathrm{~g} / \mathrm{L}$, glucose level of $22 \mathrm{mmol} / \mathrm{L}$, aspartate aminotransferase level of $834 \mathrm{U} / \mathrm{L}$, alanine aminotransferase level of $324 \mathrm{U} / \mathrm{L}$, and creatine kinase level of $234 \mathrm{U} / \mathrm{L}$, but the troponin level was less than $0.6 \mu \mathrm{g} / \mathrm{L}$. Cerebral, thoracic and abdominal computed tomographic scanning confirmed cerebral temporal contusion with edema and slight left subdural hematoma without

From the Division of General Thoracic Surgery, University Hospital, Berne, Switzerland.

Received for publication Feb 19, 2002; accepted for publication Feb 25, 2002.

Address for reprints: Thierry C. Roth, MD, Division of General Thoracic Surgery, University Hospital, 3010 Berne, Switzerland (E-mail: thierry.roth@insel.ch).

J Thorac Cardiovasc Surg 2002;124:630-1

Copyright (C) 2002 by The American Association for Thoracic Surgery

$0022-5223 / 2002 \$ 35.00+0 \quad \mathbf{1 2 / 5 4 / 1 2 4 2 6 3}$

doi: $10.1067 / \mathrm{mtc} .2002 .124263$

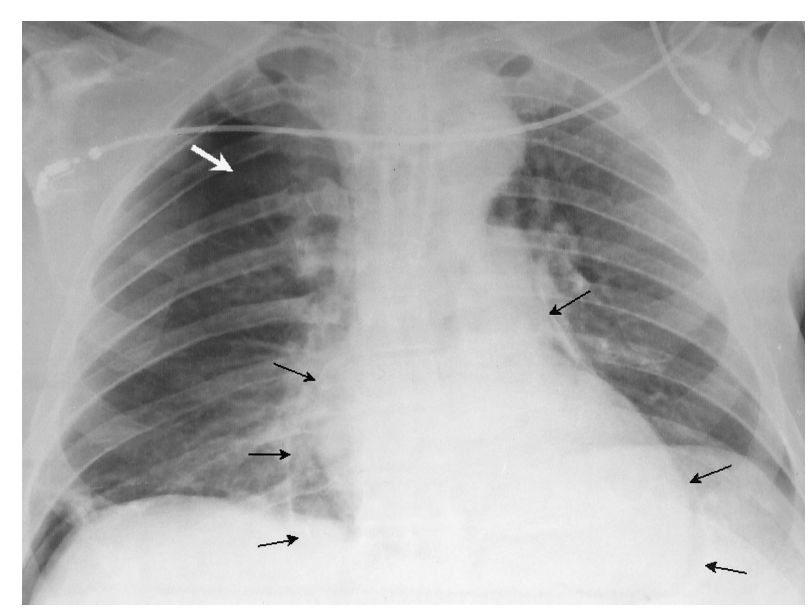

Figure 1. Chest radiograph on admission showing the presence of air in the pericardium (thin black arrows) associated with right pneumothorax (large white arrow) without rib fractures.

displacement of the median line, hematopneumothorax on the right side with bilateral lung contusions, and pneumopericardium with some pericardial fluid but no pneumomediastinum (Figure 2). In addition, a contusion of the right kidney was observed. A cystostomy was placed.

For monitoring, the patient was transferred with an insulin pump to the intensive care unit. Bronchoscopy was performed, but no bronchial laceration could be revealed. Three days after admission, a cystotomy was performed and a vesical catheter was as tutor inserted into the ruptured urethra. One day later, the patient was extubated. After removing the chest tube, atelectasis in both lungs and recurrence of right pleural effusion were observed. Evacuation was not possible with a second chest tube insertion. Seven days after admission, a right thoracoscopy was performed to remove the encapsulated fluid, and no lesion of the right side of the pericardium was visible. For the treatment of atelectasis, bronchoscopy with bronchial lavage was repeated 24 hours after reintubation. Thirteen days after admission, the last chest tube was removed, and the pneumopericardium had completely disappeared on chest radiography. One day later, the patient was discharged from our center to a peripheral hospital, and 17 days after that, he was transferred for neurorehabilitation.

\section{Discussion}

Classically, the presence of air in the pericardium occurs after a penetrating injury, principally stab wounds, and does not result in tension pneumopericardium. ${ }^{1}$ Only 32 cases of pneumopericardium after blunt trauma have been reported in the literature. ${ }^{2}$ The 3 pathogeneses proposed are direct apposition of tracheobronchial and pericardial tears, traumatic tears or congenital pleuropericardial connection that lead to pneumopericardium in association with 


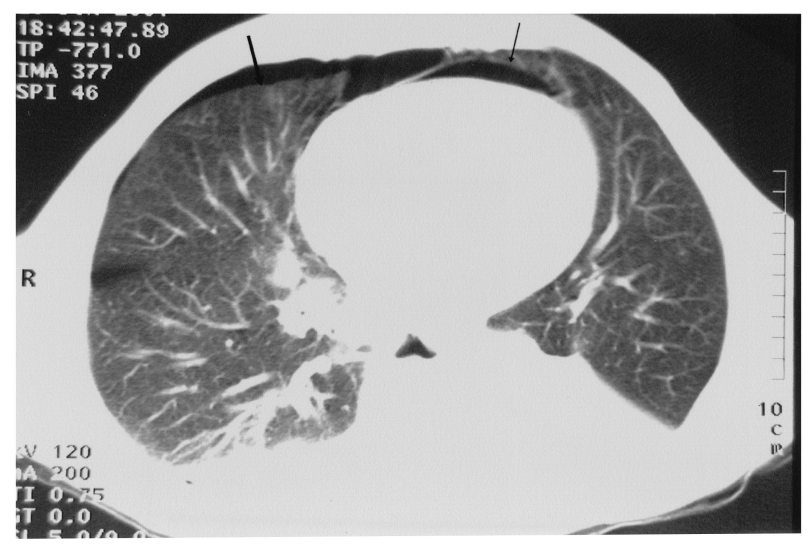

Figure 2. Chest computed tomographic scan after right-sided chest tube insertion for the pneumothorax (large arrow), which confirms the pneumopericardium (thin arrows).

a pneumothorax, and pulmonary interstitial air leaking from alveoli and tracking along the perivascular planes of the pulmonary vessels into the pericardium. ${ }^{3}$ Intubation with positive-pressure ventilation exacerbates the escape of air and might result in tension pneumopericardium. ${ }^{4}$ This situation seems to appear in $37.7 \%$ of patients, in the majority of cases in intubated patients $(83.3 \%)$, and is associated with pneumothorax in $75 \%$ of patients. ${ }^{2}$ We do not agree with some authors who advocate prophylactic pericardial decompression by means of the subxiphoid approach or percutaneous drainage to prevent potential gas tamponade ${ }^{5}$ because almost two thirds of these patients do not have this complication, and in none of the fatal cases $(41.6 \%)$ was pneumopericardium the cause of death. ${ }^{2}$ Pneumopericardium might be a sign of severe trauma, which might lead to death, and therefore monitoring in an intensive care unit is mandatory. In the majority of cases, the pneumopericardium disappears spontaneously within a few days. After blunt chest trauma in the presence of this radiologic finding, we advocate early bronchoscopy to exclude a tracheobronchial tear, which would require an immediate operative repair. Otherwise, conservative treatment is advocated.

\section{References}

1. Demetriades D, Charalambides D, Pantanowitz D, Lakhoo M. Pneumopericardium following penetrating chest injuries. Arch Surg. 1990; 125:1187-9.

2. Capizzi PJ, Martin M, Bannon MP. Tension pneumopericardium following blunt injury. J Trauma. 1995;39:775-80.

3. Westaby S. Pneumopericardium and tension pneumocardium after closed-chest injury. Thorax. 1977;32:91-7.

4. Gould JC, Schurr MA. Tension pneumopericardium after blunt chest trauma. Ann Thorac Surg. 2001;72:1728-30.

5. Gorecki PJ, Andrei VE, Schein M. Tension pneumopericardium in chest trauma. J Trauma. 1999;46:954-6. 